\title{
ON THE BOUNDARY BEHAVIOUR OF FUNCTIONS ORTHOGONAL TO INVARIANT SUBSPACES
}

BY

\author{
KAR-KOI LEUNG ${ }^{1}$
}

\begin{abstract}
In this paper we study the relationship between the boundary behaviour of functions in the orthogonal subspace of the invariant subspace $\varphi H^{2}$ and the boundary behaviour of the inner function $\varphi$ itself.
\end{abstract}

1. Introduction. Let $H^{2}$ be the Hardy space of functions analytic in the unit disc $U$ and let $S$ be the shift operator defined on $H^{2}$ by the relation

$$
S(f)=z f, \quad f \in H^{2} .
$$

It has been proved by Beurling [3] that every invariant subspace of $H^{2}$ under the shift operator $S$ is of the form $\varphi H^{2}=\left\{\varphi f: f \in H^{2}\right\}$, where $\varphi$ is an inner function, that is, a function bounded and analytic in the unit disc for which $|\varphi(z)|<1, z \in U$, and $\lim _{r \rightarrow 1}\left|\varphi\left(r e^{i \theta}\right)\right|=1$ for almost every $\theta \in(0,2 \pi]$ with respect to Lebesgue measure; see Hoffman's book [9] for details.

Let $\left\{a_{n}\right\}$ be a sequence of complex numbers such that $\sum_{n=1}^{\infty}\left(1-\left|a_{n}\right|\right)<\infty$, $0<\left|a_{n}\right|<1$. Such a sequence is known as a Blaschke sequence. The Blaschke product $B\left(z,\left\{a_{n}\right\}\right)$ associated with $\left\{a_{n}\right\}$ is defined as

$$
B\left(z,\left\{a_{n}\right\}\right)=\prod_{n=1}^{\infty} \frac{\left|a_{n}\right|}{a_{n}} \frac{a_{n}-z}{1-\bar{a}_{n} z} .
$$

It is well known that every Blaschke product $B\left(z,\left\{a_{n}\right\}\right)$ is an inner function and every inner function $\varphi$ admits the factorization

$$
\varphi(z)=c z^{m} B\left(z,\left\{a_{n}\right\}\right) \Delta(z) G(z),
$$

where $m$ is the order of zeros of $\varphi(z)$ at the origin, $\left\{a_{n}\right\}$ is the set of zeros of $\varphi$ in $U \backslash\{0\}$ repeated according to multiplicity.

Received by the editors March 13, 1974 and, in revised form, October 29, 1975 and December 16, 1976.

AMS (MOS) subject classifications (1970). Primary 30D31, 30A72, $30 \mathrm{~A} 78$.

Key words and phrases. $H^{p}$ spaces, boundary behaviour, kernel functions, orthogonal invariant subspaces, Blaschke products.

${ }^{1}$ The author wishes to express his gratitude to his supervisor, Dr. C. N. Linden, for help and useful comments during the preparation of this paper. 


$$
\begin{aligned}
& G(z)=\exp \left\{-\int_{0}^{2 \pi} \frac{e^{i \theta}+z}{e^{i \theta}-z} d \mu(\theta)\right\}, \\
& \Delta(z)=\exp \left\{-\sum_{n=1}^{\infty} \rho_{n} \frac{e^{i \delta_{n}}+z}{e^{i \delta_{n}}-z}\right\},
\end{aligned}
$$

$\mu$ is a finite, positive, continuous, singular measure defined on the unit circle $\partial U, \delta_{n} \in(0,2 \pi], \rho_{n} \geqslant 0, \sum_{n=1}^{\infty} \rho_{n}<\infty$, and $c$ is a constant of modulus one. In this paper we assume without loss of generality that $m=0$.

Let $\varphi H^{2}$ be the invariant subspace of $H^{2}$ associated with the inner function $\varphi(z)$, and let $\left(\varphi H^{2}\right)^{\perp}$ be the orthogonal complement of $\varphi H^{2}$.

For each $m>0$, each $\gamma \geqslant 1$, and each $\xi \in \partial U$, let

$$
\begin{aligned}
& R(m, \xi, \gamma)=\left\{z: 1-|z| \geqslant m|\arg z-\arg \xi|^{\gamma},\right. \\
& \qquad 0<|z|<1,|\arg z-\arg \xi| \leqslant \pi / 2\} .
\end{aligned}
$$

We say that a function $f$ defined on $U$ has a $T_{\gamma}$-limit at $\xi$ if and only if there exists a finite number $L$ such that, for each $m>0$,

$$
\lim _{\substack{z \rightarrow \xi \\ z \in R(m, \xi, \gamma)}} f(z)=L .
$$

We note [4] that $f$ has a $T_{1}$-limit at $\xi$ if and only if $f$ has a nontangential limit at $\xi$.

Ahern and Clark [2] have proved the following results concerned with the existence of $T_{1}$-limits of functions in $\left(\varphi H^{2}\right)^{\perp}$.

THEOREM A. Let $p$ be a nonnegative integer and let $\xi$ be a point of $\partial U$. Then, for each $f \in\left(\varphi H^{2}\right)^{\perp}$, the functions $f(z), f^{\prime}(z), \ldots, f^{(p)}(z)$ have $T_{1}$-limits at $\xi$ if and only if

$$
\begin{gathered}
\sum_{n=1}^{\infty} \frac{1-\left|a_{n}\right|}{\left|\xi-a_{n}\right|^{2(p+1)}}+\int_{0}^{2 \pi} \frac{d \mu(\theta)}{\left|\xi-e^{i \theta}\right|^{2(p+1)}} \\
+\sum_{n=1}^{\infty} \frac{\rho_{n}}{\left|\xi-e^{i \delta_{n}}\right|^{2(p+1)}}<\infty .
\end{gathered}
$$

Protas [13] has extended the sufficient part of this result to the case of $T_{\gamma}$-limits. In fact, he has proved

THEOREM B. Let $\gamma>1$ and let $p$ be a nonnegative integer $p<\gamma-1$. Then,

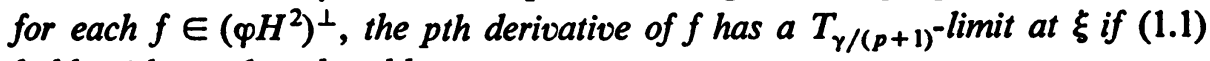
holds with $p+1$ replaced by $\gamma$.

In [13] he also gave an example to show that the converse of Theorem B is not true even for the case $p=0$ and $\varphi(z)=B\left(z,\left\{a_{n}\right\}\right)$.

The purpose of this paper is to study the boundary behaviour of the functions $f$ in $\left(\varphi H^{2}\right)^{\perp}$, and their successive derivatives along a class of curves, 
namely the curves of the form $\Gamma=\left\{r(\theta) e^{i \theta} ; \theta \in(0, \pi]\right\}$, where we assume that $r(\theta)$ is a continuous decreasing function of $\theta, 0 \leqslant r(\theta)<1, \bar{\Gamma} \cap \partial U=$ $\{1\}$.

Given a function $f$ defined on $U$, and a curve $\Gamma$ defined as above, we say that $f$ has a $\Gamma$-limit at 1 if there exists a finite complex number $L$ such that

$$
\lim _{\substack{z \rightarrow 1 \\ z \in \Gamma}} f(z)=L
$$

In $\S 2$ we obtain a sufficient condition and a necessary condition for the functions in $\left(\varphi H^{2}\right)^{\perp}$ to have $\Gamma$-limits at 1 . In $\S 3$ we study the existence of $\Gamma$-limits of higher order derivatives of functions in $\left(\varphi H^{2}\right)^{\perp}$. In $\S 4$ we study the existence of $T_{\gamma}$-limits of functions in $\left(\varphi H^{2}\right)^{\perp}$ and their derivatives. A necessary and sufficient condition is given for the case $p=0$. In $\$ 5$ an example is given to illustrate that the result we obtain in $\$ 4$ is more general than Theorem B. In $\$ 6$ we study the boundary behaviour of the kernel functions $D_{2}^{\varphi}$, which will be defined in $\$ 2$.

Of course, the results obtained in this paper are also true for the similar curves with one end at some other point on $\partial U$.

2. Let $\left\{a_{n}\right\}$ be a Blaschke sequence, and $a_{n}=r_{n} e^{i \theta_{n}}, n=1,2, \ldots$ Without loss of generality, we assume that $\left|1-a_{n}\right|<\frac{1}{2}$ for all $n$. Let $\Gamma=\left\{r(\theta) e^{i \theta}\right.$ : $\theta \in(0, \pi)\}$ be a curve defined in $U$, such that $r(\theta)$ is a continuous decreasing function of $\theta, 0<r(\theta)<1, \bar{\Gamma} \cap \partial U=\{1\}$. We have the following theorems.

THEOREM 1. Let $\varphi(z)$ be an inner function, factorized according to (1.1). If

$$
\begin{gathered}
\sum_{n=1}^{\infty} \frac{1-\left|a_{n}\right|}{\left|1-a_{n}\right|^{2}}+\int_{0}^{2 \pi} \frac{d \mu(\theta)}{\left|1-e^{i \theta}\right|^{2}}+\sum_{n=1}^{\infty} \frac{\rho_{n}}{\mid 1-e^{\left.i \delta_{n}\right|^{2}}}<\infty . \\
\varlimsup_{t \rightarrow 0^{+}}\left\{\sum_{t / 2<\theta_{n}<2 t} \frac{1-\left|a_{n}\right|}{(1-r(t))^{2}+\left(t-\theta_{n}\right)^{2}}\right. \\
\left.+\int_{t / 2}^{2 t} \frac{d \mu(\theta)}{(1-r(t))^{2}+(t-\theta)^{2}}\right\}<\infty,
\end{gathered}
$$

then, for each $f$ in $\left(\varphi H^{2}\right)^{\perp}, f(z)$ has a $\Gamma$-limit at 1 .

We mention that the range of $\left\{\theta_{n}\right\}$ considered in (2.2) has already been used [11] in studying the $\Gamma$-limits of Blaschke products $B\left(z,\left\{a_{n}\right\}\right)$, in which an associated necessary and sufficient condition is obtained for the existence of $\Gamma$-limits of the Blaschke product $B\left(z,\left\{a_{n}\right\}\right)$ and its subproducts. 
Proof. We first consider the special cases (I) $\varphi=B$, (II) $\varphi=G$, (III) $\varphi=\Delta$, and then give a proof for the general case based on the factorization (1.1).

In the case (I), let $B_{1}=1$, let $B_{n}(z)=B\left(z,\left\{a_{i}\right\}_{i=1}^{n-1}\right), n=2,3, \ldots$, and let

$$
h_{n}(z)=\left(1-\left|a_{n}\right|^{2}\right)^{1 / 2} B_{n}(z) /\left(1-\bar{a}_{n} z\right), \quad n=1,2, \ldots
$$

It was shown by Ahern and Clark [2] that $\left\{h_{n}\right\}$ is an orthonormal basis for $\left(B H^{2}\right)^{\perp}$. For any $z \in U$, let $D_{z}^{B}$ be the function in $\left(B H^{2}\right)^{\perp}$ satisfying $f(z)=\left(f, D_{z}^{B}\right)$ for all $f$ is $\left(B H^{2}\right)^{\perp}$. The function $D_{z}^{B}$ is known as the reproducing kernel of $\left(B H^{2}\right)^{\perp}$. For further details of reproducing kernels, we refer to Hille's paper [8].

We wish to show that $\left\{D_{z}^{B}: z \in \Gamma\right\}$ converges weakly as $z \rightarrow 1$ along $\Gamma$. Since $\left\{h_{n}\right\}$ is an orthonormal basis for $\left(B H^{2}\right)^{\perp}, D_{z}^{B}$ admits a representation

$$
D_{z}^{B}(\xi)=\sum_{n=1}^{\infty} \bar{h}_{n}(z) h_{n}(\xi)
$$

and

$$
\left\|D_{z}^{B}\right\|^{2}=\sum_{n=1}^{\infty}\left|h_{n}(z)\right|^{2}=\sum_{n=1}^{\infty} \frac{1-\left|a_{n}\right|^{2}}{\left|1-\bar{a}_{n} z\right|^{2}}\left|B_{n}(z)\right|^{2} .
$$

Let $z=r(t) e^{i t}$, and let $r(t)>\frac{1}{2}$. Define the functions $S_{1}, S_{2}, S_{3}$ as follows:

$$
\begin{aligned}
& S_{1}(t)=\sum_{2 t<\theta_{n}<2 \pi} \frac{1-\left|a_{n}\right|}{\left|1-\bar{a}_{n} z\right|^{2}}, \\
& S_{2}(t)=\sum_{0<\theta_{n}<t / 2} \frac{1-\left|a_{n}\right|}{\left|1-\bar{a}_{n} z\right|^{2}}, \\
& S_{3}(t)=\sum_{t / 2<\theta_{n}<2 t} \frac{1-\left|a_{n}\right|}{\left|1-\bar{a}_{n} z\right|^{2}} .
\end{aligned}
$$

Then

$$
\begin{aligned}
S_{1}(t) & =\sum_{2 t<\theta_{n}<2 \pi} \frac{1-\left|a_{n}\right|}{\left|1-\bar{a}_{n} z\right|^{2}} \\
& =\sum_{2 t<\theta_{n}<2 \pi} \frac{1-\left|a_{n}\right|}{\left(1-r_{n} r(t)\right)^{2}+4 r_{n} r(t) \sin ^{2}\left(\left(t-\theta_{n}\right) / 2\right)} \\
& <\pi^{2} \sum_{2 t<\theta_{n}<2 \pi} \frac{1-\left|a_{n}\right|}{\left|t-\theta_{n}\right|^{2}} \\
& <4 \pi^{2} \sum_{2 t<\theta_{n}<2 \pi} \frac{1-\left|a_{n}\right|}{\theta_{n}^{2}}<4 \pi^{2} \sum_{n=1}^{\infty} \frac{1-\left|a_{n}\right|}{\theta_{n}^{2}} .
\end{aligned}
$$


Similarly, we have

$$
\begin{aligned}
S_{2}(t) & <\pi^{2} \sum_{n=1}^{\infty} \frac{1-\left|a_{n}\right|}{\theta_{n}^{2}}, \\
S_{3}(t) & =\sum_{t / 2<\theta_{n}<2 t} \frac{1-\left|a_{n}\right|}{\left(1-r_{n} r(t)\right)^{2}+4 r_{n} r(t) \sin ^{2}\left(\left(t-\theta_{n}\right) / 2\right)} \\
& <\sum_{t / 2<\theta_{n}<2 t} \frac{1-\left|a_{n}\right|}{(1-r(t))^{2}+\left(t-\theta_{n}\right)^{2} / \pi^{2}} \\
& <\pi^{2} \sum_{t / 2<\theta_{n}<2 t} \frac{1-\left|a_{n}\right|}{(1-r(t))^{2}+\left(t-\theta_{n}\right)^{2}} .
\end{aligned}
$$

It has been shown by Cargo [5] that $\sum_{n=1}^{\infty}\left(1-\left|a_{n}\right|\right) /\left|1-a_{n}\right|^{p}$ converges if and only if $\sum_{n=1}^{\infty}\left(1-\left|a_{n}\right|\right) / \theta_{n}^{\rho}$ converges, where $\rho$ is a positive number. But by the hypothesis $(2.1) \sum_{n=1}^{\infty}\left(1-\left|a_{n}\right|\right) /\left|1-a_{n}\right|^{2}$ converges. Thus we have that $\sum_{n=1}^{\infty}\left(1-\left|a_{n}\right|\right) / \theta_{n}^{2}$ is convergent and hence $S_{1}(t)$ and $S_{2}(t)$ are uniformly bounded. By the hypothesis (2.2) we can find some positive constant $c$, and $\Phi \in(0, \pi]$ such that

$$
\sum_{t / 2<\theta_{n}<2 t} \frac{1-\left|a_{n}\right|}{(1-r(t))^{2}+\left(t-\theta_{n}\right)^{2}}<c, \quad t \in(0, \Phi) .
$$

Hence $\sum_{n=1}^{\infty}\left(1-\left|a_{n}\right|\right) /\left|1-\bar{a}_{n} z\right|^{2}$ is uniformly bounded on $\Gamma$ for $t \in(0, \Phi]$, and hence $\left\|D_{z}^{B}\right\|^{2}$ is uniformly bounded on $\Gamma$ for $t \in(0, \Phi]$.

Let $z$ approach 1 along $\Gamma$. Since the closed unit ball of a Hilbert space is weakly compact, there is a weak limit point of $D_{z}^{B}$ in $\left(B H^{2}\right)^{\perp}$, denoted by $D_{1}^{B}$. This weak limit point is determined by $\left(h_{n}, D_{1}^{B}\right), n=1,2, \ldots$, and each $h_{n}$ is continuous on the closure of $U$. Therefore $D_{z}^{B} \rightarrow D_{1}^{B}$ weakly as $z \rightarrow 1$ along $\Gamma$. We therefore see that Theorem 1 is true for the case $\varphi=B$.

We now consider the case $\varphi=G$. Ahern and Clark [1] have shown that the linear combinations of the projections of the $G_{\theta}$ 's into $\left(G H^{2}\right)^{\perp}$ form a dense subset of $\left(G H^{2}\right)^{\perp}$ and the projection of each $G_{\theta}$ into $\left(G H^{2}\right)^{\perp}$ can be expressed as a linear combination of the $G_{\theta}$ 's, where

$$
G_{\theta}(z)=\exp \left\{-\int_{0}^{\theta} \frac{e^{i \lambda}+z}{e^{i \lambda}-z} d \mu(\lambda)\right\}
$$

Thus we can find an orthonormal basis for $\left(G H^{2}\right)^{\perp}$ among the linear combinations of $G_{\theta}(z), \theta \in(0,2 \pi)$.

We also have for all $f \in\left(G H^{2}\right)^{\perp}$

$$
f(z)=\left(f, D_{z}^{G}\right),
$$

where 


$$
\begin{aligned}
D_{z}^{G}(\xi) & =\frac{1-\bar{G}(z) G(\xi)}{1-\bar{\xi}_{z}}, \\
\left\|D_{z}^{G}\right\|^{2} & =2 \int_{0}^{2 \pi} \frac{\left|G_{\theta}(z)\right|^{2}}{\left|1-e^{i \theta}\right|^{2}} d \mu(\theta) .
\end{aligned}
$$

From (2.4) we have for $z=r(t) e^{i t}$ and $r(t)>\frac{1}{2}$

$$
\begin{aligned}
\left\|D_{z}^{G}\right\|^{2} & <2 \pi^{2} \int_{0}^{2 \pi} \frac{d \mu(\theta)}{(1-r(t))^{2}+(t-\theta)^{2}} \\
& <5 \pi^{2} \int_{0}^{2 \pi} \frac{d \mu(\theta)}{\theta^{2}}+2 \pi^{2} \int_{t / 2}^{2 t} \frac{d \mu(\theta)}{(1-r(t))^{2}+(t-\theta)^{2}} .
\end{aligned}
$$

But $\int_{0}^{2 \pi}\left(1 / \theta^{2}\right) d \mu(\theta)$ is bounded if and only if $\int_{0}^{2 \pi}\left|1-e^{i \theta}\right|^{-2} d \mu(\theta)$ is bounded and the hypothesis (2.2) implies that there exist positive numbers $\Phi$ and $c$ such that

$$
\int_{t / 2}^{2 t} \frac{d \mu(\theta)}{(1-r(t))^{2}+(t-\theta)^{2}}<c
$$

for all $t \in(0, \Phi]$. Hence we have that $\left\|D_{z}^{G}\right\|$ is bounded uniformly for all $t \in(0, \Phi]$.

Since the closed unit ball of a Hilbert space is weakly compact, the existence of a weak limit point of $D_{z}^{G}$ in $\left(G H^{2}\right)^{\perp}$ as $z \rightarrow 1$ along $\Gamma$ is assured. We denote this weak limit point by $D_{1}^{G}$, and then $D_{1}^{G}$ is determined by $\left(G, D_{1}^{G}\right)$ when $\theta \in(0,2 \pi)$.

We now prove that $D_{1}^{G}$ is unique by showing that each $G_{\theta}$ has a $\Gamma$-limit at 1. We will say that $F$ is a divisor of $G$ if $F$ is in the unit ball of $H^{\infty}$ and there exists a function $H(z)$ in the unit ball of $H^{\infty}$ such that $G=F H$. We note that each $G_{\theta}$ is a divisor of $G$.

Now from the hypothesis (2.1), we have

$$
\int_{0}^{2 \pi} \frac{d \mu(\theta)}{\left|1-e^{i \theta}\right|}<\infty .
$$

From the hypothesis (2.2), we have

$$
\lim _{t \rightarrow 0^{+}} \int_{t / 2}^{2 t} \frac{d \mu(\theta)}{1-r(t)+|t-\theta|}=0 .
$$

But it is known [10] that (2.5) combined with (2.6) is a necessary and sufficient condition for each divisor of $G(z)$ to have a $\Gamma$-limit of modulus one at 1 . Hence we obtain the theorem for the case $\varphi=G$.

We can prove the theorem for the case $\varphi=\Delta$ by the same method as we 
used to prove the theorem for the case $\varphi=G$. Observe the fact that the linear combinations of the functions $\Delta_{\lambda}(z), \lambda \in(0, \infty)$, form a dense subset of $\left(\Delta H^{2}\right)^{\perp}[1]$, where

$$
\Delta_{\lambda}(z)=\exp \left\{-\sum_{j=1}^{N} \rho_{j} \frac{e^{i \delta_{j}}+z}{e^{i \delta_{j}}-z}-(\lambda+N) \rho_{N+1} \frac{e^{i \delta_{N+1}}+z}{e^{i \delta_{N+1}}-z}\right\},
$$

and $N=[\lambda]$ is the integral part of $\lambda$.

We have for all $f \in\left(\Delta H^{2}\right)^{\perp}$

$$
\begin{aligned}
f(z) & =\left(f, D_{z}^{\Delta}\right), \\
D_{z}^{\Delta}(\xi) & =\frac{1-\bar{\Delta}(z) \Delta(\xi)}{1-\bar{\xi}_{z}}, \\
\left\|D_{z}^{\Delta}\right\|^{2} & =2 \sum_{N=1}^{\infty} \rho_{N} \frac{\left|\Delta_{N}(z)\right|^{2}}{\mid 1-e^{\left.i \delta_{N+1}\right|^{2}}} .
\end{aligned}
$$

The proof for the case $\varphi=\Delta$ is so similar to the proof for the case $\varphi=G$ that it will be omitted. It has been observed by Ahern and Clark [1] that for linear functions $\varphi_{1}$ and $\varphi_{2}$, we have

$$
\left(\varphi_{1} \varphi_{2} H^{2}\right)^{\perp}=\left(\varphi_{1} H^{2}\right)^{\perp} \oplus \varphi_{1}\left(\varphi_{2} H^{2}\right)^{\perp} .
$$

Hence the hypotheses (2.1) and (2.2) imply that each function in $\left(B H^{2}\right)^{\perp} U$ $\left(G H^{2}\right)^{\perp} \cup\left(\Delta H^{2}\right)^{\perp}$ has a $\Gamma$-limit at 1 and the same is true for their linear combinations and finite products.

This completes the proof of Theorem 1.

Ahern and Clark [2] have shown that if all the functions in $\left(\varphi H^{2}\right)^{\perp}$ are bounded along some sequence in that unit disc, tending to 1 , then every function in $\left(\varphi H^{2}\right)^{\perp}$ has a $T_{1}$-limit at 1 . Now we can prove the following converse of Theorem 1 . In fact, we can prove something more.

THEOREM 2. Let $\varphi(z)$ be an inner function factorized according to (1.1). If for each $f$ in $\left(\varphi H^{2}\right)^{\perp}, f(z)$ is bounded along $\Gamma$ then (2.1) and (2.2) hold.

Proof. (2.1) follows easily from Theorem A.

If (2.2) were not true, then one of the following cases must arise:

$$
\begin{aligned}
\varlimsup_{t \rightarrow 0^{+}} & \left.\sum_{t / 2<\theta_{n}<2 t} \frac{1-\left|a_{n}\right|}{(1-r(t))^{2}+\left(t-\theta_{n}\right)^{2}}\right]=\infty, \\
\varlimsup_{t \rightarrow 0^{+}}\left[\int_{t / 2}^{2 t} \frac{d \mu(\theta)}{(1-r(t))^{2}+\left(1-\theta_{n}\right)^{2}}\right] & =\infty,
\end{aligned}
$$




$$
\varlimsup_{t \rightarrow 0^{+}}\left(\sum_{t / 2<\delta_{n}<2 t} \frac{\rho_{n}}{1-r(t)^{2}+\left(t-\delta_{n}\right)^{2}}\right)=\infty .
$$

In the case (I), since $\left(B H^{2}\right)^{\perp} \subset\left(\varphi H^{2}\right)^{\perp}$, we have that each function in $\left(B H^{2}\right)^{\perp}$ is bounded along $\Gamma$. Then the uniform boundedness theorem implies that $\left\|D_{z}^{B}\right\|$ is bounded for all $z \in \Gamma$, that is

$$
\left\|D_{z}^{B}\right\|^{2}=\frac{1-|B(z)|^{2}}{1-|z|^{2}}<c
$$

for some constant $c>0$. Therefore $|B(z)| \rightarrow 1$ as $z \rightarrow 1$ along $\Gamma$. Hence we can find $z_{0} \in \Gamma$ such that $|B(z)|>\frac{1}{2}$ whenever $|z|>\left|z_{0}\right|, z \in \Gamma$. Now (I) implies that

$$
\lim _{\substack{z \rightarrow 1 \\ z \in \Gamma}} \sum_{n=1}^{\infty} \frac{1-\left|a_{n}\right|}{\left|1-\bar{a}_{n} z\right|^{2}}=\infty
$$

which means that for each $z^{\prime} \in \Gamma,\left(\left|z^{\prime}\right|>\left|z_{0}\right|\right)$, and each positive number $c$, we can find $z^{\prime \prime} \in \Gamma$ with $\left|z^{\prime \prime}\right|>\left|z^{\prime}\right|$ such that

$$
\sum_{n=1}^{\infty} \frac{1-\left|a_{n}\right|}{\left|1-\bar{a}_{n} z^{\prime \prime}\right|^{2}}>4 c
$$

Hence we have

$$
\begin{aligned}
\left\|D_{z^{\prime \prime}}^{B}\right\|^{2} & =\sum_{n=1}^{\infty}\left|B_{n}\left(z^{\prime \prime}\right)\right|^{2} \frac{1-\left|a_{n}\right|^{2}}{\left|1-\bar{a}_{n} z^{\prime \prime}\right|^{2}} \\
& >\frac{1}{4} \sum_{n=1}^{\infty} \frac{1-\left|a_{n}\right|^{2}}{\left|1-\bar{a}_{n} z^{\prime \prime}\right|^{2}}>\frac{1}{4} \sum_{n=1}^{\infty} \frac{1-\left|a_{n}\right|}{\left|1-\bar{a}_{n} z^{\prime \prime}\right|^{2}}>c
\end{aligned}
$$

and (2.9) is seen to be a contradiction of (2.8). Thus (I) turns out to be impossible.

By using the same method, we can show that the cases (II) and (III) will never happen.

The theorem is thus proved.

3. We now study the existence of the $\Gamma$-limits of the higher order derivatives of functions in $\left(\varphi H^{2}\right)^{\perp}$. We have the following theorem.

THEOREM 3. Let $\varphi$ be an inner function with the factorization (1.1), and let $p$ be a nonnegative integer, if (1.2) holds with $\xi=1$, and 


$$
\begin{aligned}
\varlimsup_{t \rightarrow 0^{+}}\left\{\sum_{t / 2<\theta_{n}<2 t} \frac{1-\left|a_{n}\right|}{\left[(1-r(t))^{2}+\left(t-\theta_{n}\right)^{2}\right]^{p+1}}\right. \\
\quad+\int_{t / 2}^{2 t} \frac{d \mu(\theta)}{\left[(1-r(t))^{2}+(t-\theta)^{2}\right]^{p+1}} \\
\left.\quad+\sum_{t / 2<\delta_{n}<2 t} \frac{\rho_{n}}{\left[(1-r(t))^{2}+\left(t-\delta_{n}\right)^{2}\right]^{p+1}}\right\}<\infty .
\end{aligned}
$$

Then, for each $f \in\left(\varphi H^{2}\right)^{\perp}$, the functions $f(z), \ldots, f^{(p)}(z)$ have $\Gamma$-limits at 1 .

Proof. We first consider the special cases (I) $\varphi=B$, (II) $\varphi=G$, (III) $\varphi=\Delta$, and then give a proof for the general case.

In the case (I), by making use of (2.7) we can assume without loss of generality that $\left\{a_{n}\right\}$ satisfy $\left|1-\bar{a}_{n} z\right|<1$ for all $z \in \Gamma,|z|>\frac{1}{2}$. The theorem has been proved for the case $p=0$ in Theorem 1. We now apply the principle of mathematical induction to prove the theorem for all nonnegative integers $p$. We assume that the theorem is true for $p=N-1$. We wish to show that the theorem is also true for $p=N$.

For each $z \in \Gamma$, let $D_{z, N}^{B}$ be the function in $\left(B H^{2}\right)^{\perp}$ which satisfies

$$
f^{(N)}(z)=\left(f, D_{z, N}^{B}\right) \text {. }
$$

Since $\left\{h_{n}\right\}$ is an orthonormal basis for $\left(B H^{2}\right)^{\perp}$, we have $D_{z, N}^{B}=\sum_{n=1}^{\infty} d_{n}^{(N)} h_{n}$, where $\left\|D_{z, N}^{B}\right\|^{2}=\sum_{n=1}^{\infty}\left|d_{n}^{(N)}(z)\right|^{2}$. Setting $f=h_{n}$ we have $d_{n}^{(N)}=h_{n}^{(N)}$ and

$$
h_{n}^{(N)}(z)=\left(1-\left|a_{n}\right|^{2}\right)^{1 / 2} \sum_{j=0}^{N}\left(\begin{array}{c}
N \\
j
\end{array}\right) B_{n}^{(N-j)}(z) \frac{j ! \bar{a}_{n}^{J}}{\left(1-\bar{a}_{n} z\right)^{j+1}} .
$$

Hence

$$
\begin{aligned}
\left\|D_{z, N}^{B}\right\|^{2} & =\sum_{n=1}^{\infty}\left|d_{n}^{(N)}\right|^{2} \\
& <\sum_{n=1}^{\infty}\left(1-\left|a_{n}\right|^{2}\right)\left(\sum_{j=0}^{N}\left(\begin{array}{c}
N \\
j
\end{array}\right)\left|B_{N}^{(N-j)}(z)\right| \frac{j !\left|a_{n}\right|^{j}}{\left|1-\bar{a}_{n} z\right|^{j+1}}\right)^{2} .
\end{aligned}
$$

Now

implies that

$$
\varlimsup_{t \rightarrow 0^{+}} \sum_{t / 2<\theta_{n}<2 t} \frac{1-\left|a_{n}\right|}{\left[(1-r(t))^{2}+\left(t-\theta_{n}\right)^{2}\right]^{p+1}}<\infty
$$




$$
\varlimsup_{t \rightarrow 0^{+}} \sum_{t / 2<\theta_{n}<2 t} \frac{1-\left|a_{n}\right|}{\left[1-r(t)+\left(t-\theta_{n}\right)\right]^{p+1}}=0 .
$$

Theorem 3 of [11] asserts the boundedness of $B_{n}^{(N-j)}(z)$ on $\Gamma$ for $n=1$, $2, \ldots, j<N$, hence

$$
\frac{\left|B_{n}^{(N-j)}(z)\right| j !\left|\bar{a}_{n}\right|^{j}}{\left|1-\bar{a}_{n} z\right|^{j+1}} \leqslant \frac{c_{(N-j)} j !}{\left|1-\bar{a}_{n} z\right|^{j+1}}
$$

where $c_{(N-j)}$ is a constant independent of $n$, and we can take $z \in \Gamma,|z|>\frac{1}{2}$, then $\left|1-\bar{a}_{n} z\right|<1$ for all $n$, and hence there is a constant $c$ such that

$$
\left|h_{n}^{(N)}(z)\right|<c \frac{1-\left|a_{n}\right|}{\left|1-\bar{a}_{n} z\right|^{2(N+1)}}
$$

for all $n$, and $z$ close to 1 on $\Gamma$.

Therefore

$$
\left\|D_{z, N}^{B}\right\|^{2} \leqslant c \sum_{n=1}^{\infty} \frac{1-\left|a_{n}\right|}{\left|1-\bar{a}_{n} z\right|^{2(N+1)}} .
$$

Now, by using the method we have employed in the proof of Theorem 1, we can show that the right-hand member of (3.2) is uniformly bounded on $\Gamma$, for all $\theta \in(0, \Phi]$, where $\Phi$ is some number in $(0, \pi)$.

Again using the fact that the closed unit ball of a Hilbert space is weakly compact, we can find a weak limit point of $D_{z, N}^{B}$ in $\left(B H^{2}\right)^{\perp}$ as $z \rightarrow 1$ along $\Gamma$. We denote this weak limit point by $D_{N}^{B}$. This weak limit point is unique since it is determined by $\left(h_{n}, D_{N}^{B}\right), n=1,2, \ldots$, and each function $h_{n}$ is analytic on the closure of $U$. We have thus proved the theorem for the case $\varphi=B$ and $p=N$, and hence for all nonnegative integral values of $p$.

In the case (II) the theorem has been proved for the case $p=0$ in Theorem 1. We now apply the principle of mathematical induction to prove the theorem for all nonnegative integers $p$. We assume the theorem is true for $p=N-1$. We wish to show that the theorem is also true for $p=N$.

From the assumption that (1.2) holds with $\xi=1$ we have

$$
\int_{0}^{2 \pi} \frac{d \mu(\theta)}{\left|1-e^{i \theta}\right|^{p+1}}<\infty \text {. }
$$

From (3.1) we have

$$
\lim _{t \rightarrow 0^{+}} \int_{t / 2}^{2 t} \frac{d \mu(\theta)}{(1-r(t)+|t-\theta|)^{p+1}}=0 .
$$

But (3.3) and (3.4) imply that the $p$ th derivative of each divisor of $G$ has a $\Gamma$-limit at $1[\mathbf{1 0}]$. 
Ahern and Clark [1] have shown that for each $f \in\left(G H^{2}\right)^{\perp}$

$$
f(z)=\int_{0}^{2 \pi} c(\theta) K(\theta, z) d \mu(\theta)
$$

where $c(\theta) \in L^{2}(\mu(\theta))$, and

$$
K(\theta, z)=\frac{\sqrt{2} G_{\theta}(z)}{1-e^{-i \theta_{z}}}
$$

From (3.5) we have

$$
f^{(N)}(z)=\int_{0}^{2 \pi} c(\theta) \frac{\partial^{(N)} k}{\partial z^{N}} d \mu(\theta) .
$$

Now let $D_{z, N}^{G}$ be a function in $\left(G H^{2}\right)^{\perp}$ such that

$$
f^{(N)}(z)=\left(f, D_{z, N}^{G}\right)
$$

for each $f \in\left(G H^{2}\right)^{\perp}$. Since $L^{2}(\mu(\theta))$ is isometric to $\left(G H^{2}\right)^{\perp}$ [1], we have

$$
\left\|D_{z, N}^{G}\right\|^{2}=\int_{0}^{2 \pi}\left|\frac{\partial^{(N)} K}{\partial z^{N}}\right|^{2} d \mu(\theta)
$$

By applying Leibniz's rule to (3.6) we have

$$
\frac{\partial^{N} K(\theta, z)}{\partial z^{N}}=\sqrt{2} \sum_{n=0}^{N}\left(\begin{array}{l}
N \\
n
\end{array}\right) n ! G_{\theta}^{(N-n)}(z) \frac{\left(e^{-i \theta}\right)^{N}}{\left(1-e^{-i \theta} z\right)^{n+1}} .
$$

Since

$$
\varlimsup_{t \rightarrow 0^{+}} \int_{t / 2}^{2 t} \frac{d \mu(\theta)}{\left[(1-r(t))^{2}+(t-\theta)^{2}\right]^{p+1}}<\infty
$$

implies that (3.4) holds, by an argument similar to the proof of Theorem 1 of [10], we know that $G_{\theta}^{(N-n)}(z)$ is bounded on $\Gamma$ by $C_{n}$, where $C_{n}$ is a constant independent of $\theta$ and $z$. So there exists a constant $C$ such that

$$
\left|\frac{\partial^{N} K(\theta, z)}{\partial z^{N}}\right|^{2}<\frac{C}{\left|1-e^{-i \theta} z\right|^{2(N+1)}} .
$$

The condition (1.2) with $\xi=1$ together with condition (3.1) imply that

$$
\int_{0}^{2 \pi}\left|\frac{\partial^{N} K(\theta, z)}{\partial z^{N}}\right|^{2} d \mu(\theta)
$$

is bounded uniformly when $t \in(0, \Phi]$, where $\Phi$ is some small positive number. Hence $\left\{\left\|D_{z, N}^{G}\right\|\right\}$ is bounded uniformly on $(0, \Phi]$.

Again, we use the fact that the closed unit ball of a Hilbert space is weakly compact to find a weak limit point $D_{N}^{G}$ as $z \rightarrow 1$ along $\Gamma$. But $D_{z}^{G}$ is 
determined by $\left(G_{\theta}, D_{N}^{G}\right), \theta \in(0,2 \pi]$, and the existence of the $\Gamma$-limit of each $G_{\theta}^{(N)}$ ensures that $D_{N}^{G}$ is indeed the weak limit of $D_{z, N}^{G}$ as $z \rightarrow 1$ along $\Gamma$. This completes the proof for that case $\varphi=G$.

The proof for the case $\varphi=\Delta$ is very similar to the proof for the case $\varphi=G$.

Combining the above results for the cases $\varphi=B, \varphi=G$, and $\varphi=\Delta$, and observing the relation (2.7) we now see that the theorem is true for $p=N$, and for all nonnegative integral values of $p$.

4. We now apply the results obtained in $\$ \S 2$ and 3 to study the $T_{\gamma}$-limit of functions in the space $\left(\varphi H^{2}\right)^{\perp}$. We first obtain

THEOREM 4. Let $\varphi(z)$ be an inner function, let (1.1) be its factorization, and let $\gamma>1$. Then for each function $f$ in $\left(\varphi H^{2}\right)^{\perp}, f(z)$ has a $T_{\gamma}$-limit at 1 if and only if (1.2) holds with $\xi=1, p=0$ and

$$
\begin{aligned}
\varlimsup_{t \rightarrow 0^{+}}\left\{\sum_{t / 2<\left|\theta_{n}\right|<2 t} \frac{1-\left|a_{n}\right|}{t^{2 \gamma}+\left(t-\left|\theta_{n}\right|\right)^{2}}\right. \\
\quad+\left(\int_{-2 t}^{-t / 2}+\int_{t / 2}^{2 t}\right) \frac{d \mu(|\theta|)}{t^{2 \gamma}+(t-|\theta|)^{2}} \\
\left.\quad+\sum_{t / 2<\left|\delta_{n}\right|<2 t} \frac{\rho_{n}}{t^{2 \gamma}+\left(t-\left|\delta_{n}\right|\right)^{2}}\right\}<\infty .
\end{aligned}
$$

Proof. We obtain the sufficiency if (1.2) with $\xi=1, p=0$ and (4.1) by noticing the fact that the modulus of the value of each function in $\left\{h_{n}\right\} \cup$ $\left\{G_{\theta}\right\} \cup\left\{\Delta_{\lambda}\right\}$ in each set $R(m, 1, \gamma)$ for $m>0$ is not greater than the modulus of its values on the boundary curve $r(t)=1-m|t|^{\gamma}$, and applying Theorem 1.

In considering the necessary condition, we note that (1.2) with $\xi=1, p=0$ is obtained by applying Theorem $A$ and the fact that the existence of a $T_{\gamma}$-limit of a function defined on $U$ for some $\gamma>1$ at a point $\xi \in \partial U$ implies the existence of a $T_{1}$-limit of this function at the same point $\xi$. The condition (4.1) is obtained by applying Theorem 2 to the curve defined by

$$
r(t)=1-\pi^{-\gamma}|t|^{\gamma}, \quad t \in(0, \pi] .
$$

COROLLARY 1. Let $\gamma>1$. The following statements are equivalent:

(I) each function $f \in\left(\varphi H^{2}\right)^{\perp}$ has a $T_{\gamma}$-limit at 1 ,

(II) each function $f \in\left(\varphi H^{2}\right)^{\perp}$ is bounded along $R(m, 1, \gamma)$.

As an application of Theorem 3, we have the following result:

THEOREM 5. Let $\varphi$ be an inner function, and let (1.1) be its factorization. Let $\gamma>1$, and let $p$ be a nonnegative integer. If (1.2) holds with $\xi=1$ and 


$$
\begin{aligned}
\varlimsup_{t \rightarrow 0^{+}}\left\{\sum_{t / 2<\left|\theta_{n}\right|<2 t} \frac{1-\left|a_{n}\right|}{\left(t^{2 \gamma}+\left(t-\left|\theta_{n}\right|^{2}\right)\right)^{p+1}}\right. \\
+\left(\int_{-2 t}^{-t / 2}+\int_{t / 2}^{2 t}\right) \frac{d \mu(|\theta|)}{\left(t^{2 \gamma}+(t-|\theta|)^{2}\right)^{p+1}} \\
\left.+\sum_{t / 2<\left|\delta_{n}\right|<2 t} \frac{\rho_{n}}{\left(t^{2 \gamma}+\left(t-\left|\delta_{n}\right|\right)^{2}\right)^{p+1}}\right\}<\infty
\end{aligned}
$$

then the pth derivative of each $f \in\left(\varphi H^{2}\right)^{\perp}$ has a $T_{\gamma}$-limit at 1 .

Note. (1) If the $p$ th derivative of each $f \in\left(\varphi H^{2}\right)^{\perp}$ has a $T_{\gamma}$-limit at 1 then the $j$ th derivative of each $f \in\left(\varphi H^{2}\right)^{\perp}$ will have a $T_{\lambda_{j}}$-limit for $\lambda_{j}=(p+$ 1) $\gamma /(j+1)$ at $1, j=0,1, \ldots, p$.

(2) It is easily seen that as long as we can prove the existence of the limit of the $p$ th derivative of each $f \in\left(\varphi H^{2}\right)^{\perp}$ as $z \rightarrow 1$ in $R(m, 1, \gamma)$ for some particular $m>0$, by this method we can also prove the existence of the limit of each $f \in\left(\varphi H^{2}\right)^{\perp}$ as $z \rightarrow 1$ in $R(M, 1, \gamma)$ for any other $M>0$.

5. In this section, we give an example to show that indeed Theorem 5 is more general than Theorem B.

EXAMPLE. Let $p$ be a positive integer and $\gamma>1$. Then there exists a Blaschke sequence $\left\{a_{n}\right\}$ such that

$$
\sum_{n=1}^{\infty} \frac{1-\left|a_{n}\right|}{\left|1-a_{n}\right|^{\lambda(2 p+2)}}=\infty
$$

whenever

$$
\lambda>\frac{1+\gamma(2 p+1)}{2 p+2}
$$

but the $p$ th derivative of each function $f$ in $\left(B H^{2}\right)^{\perp}$ has a $T_{\gamma}$-limit at 1 . Let

$$
a_{n}=\left(1-\frac{1}{n^{\beta} \log n}\right) e^{i / n^{\alpha}}, \quad n=2,3, \ldots,
$$

where we assume that

$$
\beta>\max (1, \alpha), \quad 0<\alpha<1 /(\gamma-1) .
$$

Then

$$
\frac{1-\left|a_{n}\right|}{\left|1-a_{n}\right|^{\lambda(2 p+2)}} \sim \frac{n^{\alpha(2 p+2) \lambda-\beta}}{\log n}
$$

as $n \rightarrow \infty$, so that (5.1) holds if and only if

$$
\alpha(2 p+2) \lambda-\beta>1 \text {. }
$$


But we require our Blaschke sequence to satisfy (1.2) with $\xi=1$. For this purpose, we let

$$
\alpha(2 p+2) \lambda-\beta<-1
$$

We now impose some conditions on the Blaschke sequence (5.1) so that (4.2) can be satisfied. Let $a_{n}=r_{n} e^{i \theta_{n}}$. For small positive values of $t$, we define $N$ so that

$$
(N+1)^{-\alpha}<t<N^{-\alpha}
$$

Let $K$ be a positive number less than one. Then for some constant $C$ we have

$$
\sum_{|n-N|<N^{K}} \frac{1-r_{n}}{\left\{t^{\gamma}+\left|t-\theta_{n}\right|\right\}^{2 p+2}}<\frac{C N^{K+\alpha \gamma(2 p+2)-\beta}}{\log N}
$$

while the remaining terms of (4.2) are bounded by

$$
\sum_{N^{K}<|n-N|<C N} \frac{C N^{(\alpha+1)(2 p+2)-\beta}}{|N-n|^{2 p+2} \log N}<\frac{C N^{(\alpha+1)(2 p+2)-K(2 p+1)-\beta}}{\log N} .
$$

Hence (4.2) will be satisfied if both of the following inequalities hold

$$
\begin{gathered}
K+\alpha \gamma(2 p+2)-\beta<0, \\
(\alpha+1)(2 p+2)-K(2 p+1)-\beta<0 .
\end{gathered}
$$

We now see that the set of triples $(K, \alpha, \beta)$ which satisfies (5.2), (5.3), (5.4), (5.5), (5.6) is nonempty, indeed the triple

$$
K=1-\frac{\gamma-1}{[(2 p+1) \gamma+1](2 p+2)}
$$

and

$$
\begin{aligned}
& \alpha=\frac{1}{[(2 p+1) \gamma+1](2 p+2)}, \\
& \beta=1+\frac{1}{2 p+2},
\end{aligned}
$$

can meet our need.

6. The reproducing kernel $D_{z}^{\varphi}(\xi)=(1-\overline{\varphi(z)} \varphi(\xi)) /\left(1-\bar{\xi}_{z}\right)$ has been seen to be significant in the theory presented in the previous sections. Perhaps it is natural to ask whether there is any information about the inner function $\varphi$ implied by the boundary behaviour of $D_{z}^{\varphi}$ on the curve $\Gamma$. Protas [14] has obtained the following results for the curves $\Gamma_{(1, \gamma, m)}=\left\{r(t) e^{i t}, r(t)=1-\right.$ $\left.m|t|^{\gamma}, 0<|t|<\min \left(\pi, m^{-1 / \gamma}\right)\right\}$ where $\gamma>1$ and $m>0$.

THEOREM C. Let $\varphi(z)$ be an inner function, and let (1.1) be its factorization. Then (1.2) holds with $2(p+1)$ replaced by $\gamma$ if and only if 


$$
\int_{\Gamma(1, \gamma, m)}\left\|D_{z}^{\Phi}\right\|^{2}|d z|<\infty
$$

As a by-product of the proof of Theorem $\mathrm{C}$, he has also obtained

THEOREM D. If the condition (1.2) holds with $2(p+1)$ replaced by $\gamma$, then

$$
\int_{\Gamma(1, \gamma, m)}\left|\varphi^{\prime}(z)\right||d z|<\infty .
$$

Let $\Gamma=\left\{r(\theta) e^{i \theta}, \theta \in(0, \pi)\right\}$ where $r(\theta)<1$ and $\lim _{\theta \rightarrow 0} r(\theta)=1$, we assume that $r(\theta)$ satisfies a Lipschitz condition.

$$
\sup _{\theta_{1} \neq \theta_{2}}\left|r\left(\theta_{1}\right)-r\left(\theta_{2}\right)\right|<Q\left|\theta_{1}-\theta_{2}\right|
$$

and $r^{\prime}(\theta)<0$. Let $\Gamma_{1}$ be the reflexion of $\Gamma$ in the lower half-plane and let $\Gamma^{*}=\Gamma \cup \Gamma_{1}$. By using the method of Protas [14], we can prove the following extension of Theorem $\mathrm{C}$ to the curve $\Gamma$.

THEOREM 6. Let $\varphi(z)$ be an inner function, and let (1.1) be its factorization. Let $\Gamma^{*}$ be the curve defined above. Then

$$
\sum_{n=1}^{\infty} \frac{1-\left|a_{n}\right|}{1-r\left(\left|\theta_{n}\right|\right)}+\int_{-\pi}^{\pi} \frac{d \mu(|\theta|)}{1-r(|\theta|)}+\sum_{n=1}^{\infty} \frac{\rho_{n}}{1-r\left(\left|\delta_{n}\right|\right)}<\infty
$$

if and only if

$$
\int_{\Gamma^{*}}\left\|\Delta_{z}^{\Phi}\right\|^{2}|d z|<\infty
$$

Proof. We first consider the case $\varphi=B$.

From (2.3) we have, for any positive constant $C$,

$$
\int_{0}^{C}\left\|D_{r}^{B}(t) e^{i t}\right\|^{2} d t<\sum_{n=1}^{\infty} z\left(1-\left|a_{n}\right|\right) \int_{0}^{C} \frac{d t}{\| 1-\left.\bar{a}_{n} r(t) e^{i t}\right|^{2}} .
$$

Since, when $z=r(t) e^{i t}$, we have

$$
\left|1-\bar{a}_{n} z\right|^{2}>(1-r(t))^{2}+\frac{1}{2}\left|t-\theta_{n}\right|^{2}
$$

then, for sufficiently small positive numbers $c^{\prime}$

$$
\begin{aligned}
& \int_{0}^{c^{\prime}} \frac{d t}{\left|1-\bar{a}_{n}(t) e^{i t}\right|^{2}} \\
& \quad<\int_{0}^{\theta_{n}-A_{n}} \frac{2 d t}{\left(t-\theta_{n}\right)^{2}}+\int_{\theta_{n}-A_{n}}^{\theta_{n}+A_{n}} \frac{d t}{(1-r(t))^{2}}+\int_{\theta_{n}+A_{n}}^{c^{\prime}} \frac{2 d t}{\left(t-\theta_{n}\right)^{2}} \\
& \quad<\frac{2}{A_{n}}-\frac{2}{\theta_{n}}+\frac{2 A_{n}}{\left[1-r\left(A_{n}\right)\right]^{2}}+\frac{2}{A_{n}}-\frac{2}{c^{\prime}-\theta_{n}},
\end{aligned}
$$

where $A_{n}=\left(1-r\left(\theta_{n}\right)\right) /(Q-1), n=1,2, \ldots$ But, from the hypothesis 
(6.2), we have

$$
1-r\left(\theta_{n}-A_{n}\right)>\left(1-r\left(\theta_{n}\right)\right)-Q A_{n}=A_{n},
$$

and hence

$$
\int_{0}^{c^{\prime}} \frac{d t}{\left|1-\bar{a}_{n} r(t) e^{i t}\right|^{2}}=o\left(\frac{1}{1-r\left(\theta_{n}\right)}\right), \quad \theta_{n} \rightarrow 0 .
$$

It follows from (6.3) that

$$
\int_{0}^{C}\left\|D_{r(t) e^{l l}}^{B}\right\|^{2} d t<\infty
$$

and

$$
\int_{-C}^{0} \| D_{r(|t|) e^{i t} \|^{2} d t<\infty}
$$

follows in the same way.

We now see that (6.4) follows from (6.5) and (6.6), since $r^{\prime}(t)$ is bounded by $Q$.

For the converse, we assume that (6.4) holds for $\varphi=B$. By considering the logarithmic derivative of $B\left(z,\left\{a_{n}\right\}\right)$ we have

$$
B^{\prime}\left(z,\left\{a_{n}\right\}\right)=-\sum_{n=1}^{\infty} b_{n}(z) \frac{1-\left|a_{n}\right|^{2}}{\left(1-\bar{a}_{n} z\right)^{2}}
$$

where

$$
b_{n}(z)=B\left(z,\left\{a_{n}\right\}\right)\left(1-\bar{a}_{n} z\right) /\left(a_{n}-z\right), \quad n=1,2, \ldots
$$

Let $D(z)=B\left(z,\left\{a_{n}\right\}\right)^{2}$. Then $\left\|D_{z}^{B}\right\|^{2}>\left|B\left(z,\left\{a_{n}\right\}\right)\right|,\left|B^{\prime}\left(z,\left\{a_{n}\right\}\right)\right|$ $>\frac{1}{2}\left|D^{\prime}(z)\right|$, and hence

$$
\int_{\Gamma^{*}}\left|D^{\prime}(z)\right||d z|<\infty
$$

But (6.7) implies that the Blaschke product $D(z)$ has a $\Gamma$-limit $\left(\Gamma_{1}\right.$-limit) at 1. Also (6.4) implies that

$$
\int_{\Gamma^{*}} \frac{1-|D(z)|}{1-|z|}|d z|<\infty \text {. }
$$

Then, for some constant $C>0$

$$
\int_{0}^{C} \frac{1-\left|D\left(r(t) e^{i t}\right)\right|}{1-r(t)} d t<\infty .
$$

Since $r^{\prime}(t)$ is bounded for $t \in(0, C]$, hence $\sup \{|D(z)|: z \in \Gamma\}=1$ and $|D(z)| \rightarrow 1$ as $z \rightarrow 1$ along $\Gamma$. Therefore $D(z)$ has a $\Gamma$-limit of modulus 1 , and hence $B(z)$ has a $\Gamma$-limit of modulus 1 at 1 . The same is true for the curve $\Gamma_{1}$. 
Now, from (6.4), we have

$$
\int_{-C}^{C} \sum_{n=1}^{\infty} \frac{1-\left|a_{n}\right|^{2}}{\left|1-\bar{a}_{n} r(t) e^{i t}\right|^{2}} d t<\infty,
$$

and this implies that

$$
\sum_{n=1}^{\infty}\left(1-\left|a_{n}\right|^{2}\right) \int_{I_{n}}\left|1-\bar{a}_{n} r(|t|) e^{i t}\right|^{-2} d t<\infty,
$$

where $I_{n}=\left(\theta_{n}, \theta_{n}+\left(1-r\left(\theta_{n}\right)\right)\right)$ if $\theta_{n}>0$ and $I_{n}=\left(\theta_{n}-\left(1-r\left(\left|\theta_{n}\right|\right)\right), \theta_{n}\right)$ if $\boldsymbol{\theta}_{n}<0$.

Now, a theorem of Lindelöf $\left[6\right.$, p. 5] shows that $B\left(z,\left\{a_{n}\right\}\right)$ has a limit of modulus one as $z \rightarrow 1$ inside the area bounded by $\Gamma^{*}$. So there is an integer $n_{0}$ such that $a_{n}$ is outside of $\Gamma^{*}$ for each $n>n_{0}$, that is

$$
1-r_{n}<1-r\left(\left|\theta_{n}\right|\right) \text { if } n>n_{0} \text {. }
$$

Then

$$
\left|1-\bar{a}_{n} r(|t|) e^{i t}\right|^{2}<5\left(1-r\left(\left|\theta_{n}\right|\right)\right)^{2}
$$

so

$$
\sum_{n=n_{0}}^{\infty} \frac{1-\left|a_{n}\right|}{1-r\left(\left|\theta_{n}\right|\right)}<\infty
$$

and hence

$$
\sum_{n=1}^{\infty} \frac{1-\left|a_{n}\right|}{1-r(|\theta|)}<\infty
$$

In the case $\varphi=G$ and $\varphi=\Delta$, using the same method we can prove that Theorem 6 is true. Since the proof is so similar to the proof for $\varphi=B$. We will not repeat the arguments here.

Now consider that general case that $\varphi=c B G \Delta$. If $\Phi$ and $\Psi$ are two inner functions we have

$$
1-|\Phi(z) \Psi(z)|^{2}=1-|\Phi(z)|^{2}+|\Phi(z)|^{2}\left(1-|\Psi(z)|^{2}\right),
$$

and hence we have

$$
\left\|D_{z}^{\Phi \Psi}\right\|^{2}=\left\|D_{z}^{\Phi}\right\|^{2}+|\Phi(z)|^{2}\left\|D_{z}^{\Psi}\right\|^{2}<\left\|D_{z}^{\Phi}\right\|^{2}+\left\|D_{z}^{\Psi}\right\|^{2} \text {. }
$$

Combining the results we obtained for the cases $\varphi=\nabla, \varphi=G$ and $\varphi=\Delta$, we see that (6.4) implies (6.3).

Conversely, let us assume that (6.3) holds. Since $1-|\Phi(z) \Psi(z)|^{2}>1-$ $|\Phi(z)|^{2}$ for any inner function $\Psi$ and $\Phi$, we have

$$
\left\|D_{z}^{\Phi \Psi}\right\|^{2}>\left\|D_{z}^{\Phi}\right\|^{2}
$$


Again, combining the results obtained for the cases $\varphi=B, \varphi=G$ and $\varphi=\Delta$, we see that (6.3) implies (6.4).

This completes the proof of Theorem 6.

As an extension of Theorem $\mathrm{D}$, we mention the following theorem.

THEOREM 7. Let $\varphi$ be an inner function, and let (1.1) be its factorization. Then (6.3) implies

$$
\int_{\Gamma}\left|\varphi^{\prime}(z)\right||d z|<\infty
$$

\section{REFERENCES}

1. P. R. Ahern and D. N. Clark, On functions orthogonal to invariant subspaces, Acta Math. 124 (1970).

2. , Radial limits and invariant subspaces, Amer. J. Math. 92 (1970).

3. A. Beurling, On two problems concerning linear transformations in Hilbert spaces, Acta Math. 81 (1949).

4. G. T. Cargo, The radial images of Blaschke products, J. London Math. Soc. 36 (1961).

5. Angular and tangential limits of Blaschke products and their successive derivatives, Canad. J. Math. 14 (1962).

6. M. L. Cartwright, Integral functions, Cambridge Tract, no. 44, 1956.

7. D. Frostman, Sur les produits de Blaschke, Kungliga Fysiografiska Sällskapets i Lund Förhandlingar, Band 12, 1942.

8. E. Hille, Introduction to general theory of reproducing kernels, Rocky Mountain J. Math. 2 (1972), 321-368.

9. K. Hoffman, Banach spaces of analytic functions, Prentice-Hall, Englewood Cliffs, N.J., 1962.

10. Kar-Koi Leung, Asymptotic values of modulus 1 of functions in the unit ball of $H^{\infty}$, Trans. Amer. Math. Soc. 203 (1975), 119-128.

11. K. K. Leung and C. N. Linden, Asymptotic values of modulus 1 of Blaschke products, Trans. Amer. Math. Soc. 203 (1975), 107-118.

12. D. Protas, Tangential limits of Blaschke products and functions of bounded characteristics, Arch. Math. 22 (1972).

13. Tangential limits of functions orthogonal to invariant subspaces, Trans. Amer. Math. Soc. 166 (1972), 163-172.

14. , On the accumulation of the zeros of a Blaschke product at a boundary point, Proc. Amer. Math. Soc. 34 (1972), 489-496.

Current address: 22 Fa Po Street, Flat B2 3rd Floor, Kowloon, Hong Kong 